\title{
AGENTES RELACIONADOS A INFECCIÓN DE SITIO OPERATORIO EN ADULTOS MAYORES POS OPERADOS EN EL CENTRO MÉDICO NAVAL, 2013 - 2017
}

AGENTS RELATED TO AN OPERATIVE SITE INFECTION IN OPERATED OLDER ADULTS AT THE NAVAL MEDICAL CENTER, 2013 - 2017

Lucía Fernanda Hidalgo-Vizarreta', Magdiel José Manuel Gonzales-Menéndez ${ }^{2, a, c}$, Cecilia Roxana Salinas-Salas ${ }^{2 a, b}$

\begin{abstract}
RESUMEN
Introduccion: Las Infecciones de Sitio Operatorio (ISO) son parte del grupo de infecciones intrahospitalarias más frecuentes en el posoperatorio de los pacientes adultos mayores. Objetivo: Determinar los agentes relacionados a infecciones del sitio operatorio en pacientes adulto mayores pos operados en el Centro Medico Naval "Cirujano Mayor Santiago Távara" (CEMENA) de enero 2013 a diciembre 2017. Métodos: Estudio de diseño casos y controles, analítico y observacional. Se estudiaron las variables ISO como desenlace e hipertensión arterial, diabetes mellitus, neoplasia maligna, obesidad, técnica quirúrgica y tipo de cirugía como agentes. Resultados: De los 219 adultos mayores, el 33,33 \% ( $n=73)$ tuvieron ISO. En el análisis bivariado se encontraron asociaciones estadísticamente significativas para diabetes mellitus (OR: 1,49, IC 1,03-2,18, $p<0,035$ ), tipo de cirugía (OR: 4,63 IC 2,89-7,42, $p<0,05)$ y técnica quirúrgica $(O P, 0,24, I C \quad 0,13-0,43, p<0,05)$. En el análisis multivariado, se encontró que la cirugía de emergencia tiene 4,04 (OR 4,04, IC 2,55-6,40, p<0,05) veces la probabilidad de ISO en comparación a la cirugía programada, y la cirugía laparoscópica tiene 0,29 (OP 0,29 , IC $0,17-0,52, p<0,05$ ) veces la probabilidad de ISO en comparación con la técnica abierta. Conclusión: La técnica operatoria laparoscópica disminuye la probabilidad de ISO, y la cirugía de emergencia aumenta su probabilidad en pacientes adultos mayores.
\end{abstract}

Palabras clave: Adulto mayor; Infección de sitio operatorio; Cirugía. (fuente: DeCS BIREME)

\section{ABSTRACT}

Introduction: Surgical site infections (SSI) are part of the most frequent intrahospital infections in the postoperative period of elderly patients. Objective: To determine the agents related to infections of the operative site in the elderly patients after surgery of the Naval Medical Center during January 2013 to December. Methods: An analytical cross sectional study using a secondary data analysis from clinical records of patients older than 65 years post-operated. SSI was studied has outcome, and arterial hypertension, diabetes mellitus, malignant neoplasia, obesity, surgical technique and type of surgery were the agents. Results: Of the 219 older adults, 33,33\% ( $n=73$ ) had SSI. In the bivariate analysis, statistically significant associations were found for diabetes mellitus (PR: $1,49, \mathrm{Cl}$ $1,03-2,18, p<0,035$ ), type of surgery (PR: 4,63 IC 2,89-7,42, p <0,05) and surgical technique (PR.0,24, $\mathrm{Cl} 0,13-0,43, \mathrm{p}<0,05)$. In the multivariate analysis, it was found that emergency type surgery has 4,04 (PR 4,04, IC 2,55 - 6,40, p <0,05) times chance for SSI compared to the programmed surgery, and the laparoscopic technique surgery has 0,29 (PR 0,29, Cl 0,17-0,52, $\mathrm{p}<0,05$ ) chance of SSI compared to the open technique. Conclusion: Laparoscopic operative technique decreases the likelihood of ISO, and emergency surgery increases its likelihood in elderly patients.

Key words: Elderly; Surgical Site infection; Type of surgery; Surgery. (source: MeSH NLM)

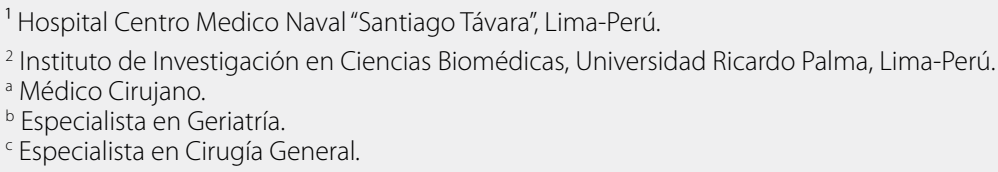

Citar como: Lucía Fernanda Hidalgo-Vizarreta, Magdiel José Manuel Gonzales-Menéndez, Cecilia Roxana Salinas-Salas. Agentes relacionados a infección de sitio operatorio en adultos mayores pos operados en el centro médico naval, 2013 - 2017. Rev. Fac. Med. Hum. Julio 2019; 19(3):43-47. DOI 10.25176/RFMH.v19i3.2163 


\section{INTRODUCCIÓN}

Las Infecciones de Sitio Operatorio (ISO) son la segunda causa más común de infecciones intrahospitalarias en los Centros de Salud' ocasionando un mayor tiempo de hospitalización, incapacidad y secuelas en el paciente con un obvio impacto económico'. Éstas aparecen generalmente dentro de los 30 días siguientes a la intervención quirúrgica, siendo comúnmente entre el día cinco y diez del posoperatorio ${ }^{1,2}$ En los hospitales de los Estados Unidos se estima que anualmente se producen cerca de 300000 de ISO, dando lugar a varios miles de millones de dólares en costos médicos potencialmente evitables ${ }^{2}$ y se estima que entre el 40 al $60 \%$ de las ISO en el mundo son prevenibles ${ }^{2,3} \mathrm{En}$ el Perú, entre enero del 2013 y diciembre del 2014 se notificó al sistema de vigilancia epidemiológica de infecciones intrahospitalarias (SVEIIH) que las ISO se incrementaron de 214 a 249 siendo el 77,5\% ( $n=190)$ reportado en los gobiernos regionales y en el Instituto de Gestión de Servicios de Salud (IGSS), el 9,6\% ( $n=24)$ en establecimientos de salud privados, el $8,8 \%(n=22)$ en Essalud, el 3,2\% ( $n=8)$ en las Fuerzas Armadas y Policiales y en los $0,8 \%(n=2)$ en los gobiernos local y mixto 4 .

El proceso del envejecimiento de los seres humanos, es un proceso natural, progresivo e irreversible que a nivel mundial en los últimos años, está sujeto a un aumento de la esperanza de vida y por lo tanto debe estar sujeto a una mejor calidad de vida. Esta población se caracteriza por tener un patrón de comorbilidades, las cuales desde el punto de vista quirúrgico son determinantes para el surgimiento de algunas complicaciones durante su estancia hospitalaria completa. Identificar estas comorbilidades y condiciones asociadas permitirán disminuir tanto las complicaciones intrahoperatorias como las postoperatorias para lograr no solo un buen acto quirúrgico, sino uno exitoso ${ }^{5,6}$.

\section{MÉTODOS}

Estudio de diseño de casos y controles, analítico y observacional, mediante análisis de datos secundarios a partir de una base generada por el CEMENA en el Servicio de Cirugía. Se contó con una muestra de 219 pacientes del Servicio de Cirugía del CEMENA; operados entre el 2013 y 2017, con un muestreo probabilístico. Se estableció como variable dependiente ISO. Las variables independientes fueron género, obesidad, grado militar, hipertensión arterial, diabetes mellitus (DM), neoplasia maligna, tipo de cirugía y técnica quirúrgica. En el análisis estadístico se utilizaron modelos predictivos de tipo regresión logística y modelos lineales generalizados con un nivel de significancia estadística de $p<0,05$ utilizando el programa Stata 14 para Windows (StataCorp LP, College Station, TX, USA).

\section{RESULTADOS}

Se contó con una muestra de 219 adultos mayores, de los pacientes con ISO, 33,08\% $(n=43)$ fueron del sexo masculino y $33,71 \%(n=30)$ del sexo femenino, $66,73 \%$ $(n=72)$ tuvieron grado militar (subalterno, oficial); además, 39,13\% ( $n=36)$ hipertensión arterial, 41,05\% $(n=39)$ DM, 34,78\% $(n=16)$ manifestó alguna neoplasia maligna, mientras que $32,05 \%(n=25)$ tuvo obesidad. Por otro lado, 56 adultos mayores presentaron ISO posterior a una cirugía de emergencia, lo cual representa el $61,54 \%$ del total de la muestra y el $48,09 \%(n=63 \%)$ del total presentaron esta misma complicación después de una cirugía realizada con técnica abierta. Para datos complementarios, revisar la tabla 1.

Respecto del análisis bivariado de ISO, mediante el uso de la regresión logística se encontraron asociaciones estadísticamente significativas p ara D M (OR: 1,49 , IC $1,03-2,18, p<0,035$ ), tipo de cirugía (OR: 4,63 IC 2,89$7,42, p<0,05)$ y técnica quirúrgica (OR:0,24, IC 0,13$0,43, p<0,05)$. Para datos adicionales, revisar la tabla 2 .

Respecto del análisis multivariado, se analizó la variable ISO ajustada por las variables estadísticamente significativas del análisis bivariado (DM, tipo de cirugía y técnica quirúrgica). Luego del análisis se encontraron asociaciones con el tipo de cirugía (OR: 4,04, IC $2,55-6,40, p<0,05$ ) y técnica quirúrgica (OR: 0,29 , IC $0,17-0,52, p<0,05)$. Esto se interpreta de la siguiente manera: la Cirugía de emergencia tiene 4,04 veces la probabilidad de ocurrencia de ISO en comparación con la cirugía programada, y la cirugía de técnica laparoscópica tiene 0,29 veces la probabilidad de ISO en comparación con la técnica abierta. Para datos adicionales, revisar la tabla 3. 
Tabla 1. Características poblacionales y quirúrgicas según infección de sitio operatorio.

\begin{tabular}{lcc}
\hline \multicolumn{2}{c}{ Infección de sitio operatorio } \\
Sexo & No & Si \\
Femenino & $59(66,29 \%)$ & $30(33,71 \%)$ \\
Masculino & $87(66,92 \%)$ & $43(33,08 \%)$ \\
Grado militar & & \\
Subalterno & $84(70 \%)$ & $36(30 \%)$ \\
Oficial & $62(63,27 \%)$ & $36(36,73 \%)$ \\
Civil & 0 & $1(100 \%)$ \\
Hipertensión arterial & & \\
No & $90(70,87 \%)$ & $37(29,13 \%)$ \\
Si & $56(60,87 \%)$ & $36(39,13 \%)$ \\
Diabetes mellitus & & $34(27,42 \%)$ \\
No & $90(72,58 \%)$ & $39(41,05 \%)$ \\
Si & $56(58,95 \%)$ & \\
Neoplasia maligna & & $57(32,95 \%)$ \\
No & $116(67,05 \%)$ & $16(34,78 \%)$ \\
Si & $30(65,22 \%)$ & \\
Obesidad & & $48(34,04 \%)$ \\
No & $93(65,96 \%)$ & $25(32,05 \%)$ \\
Si & $53(67,95 \%)$ & $17(13,28 \%)$ \\
Tipo de cirugía & & $56(61,54 \%)$ \\
Programada & $111(86,72 \%)$ & \\
Emergencia & $35(38,46 \%)$ & $63(11,36 \%)$ \\
\hline Técnica quirúrgica & $68(51,91 \%)$ & \\
Abierta & $78(88,64 \%)$ & \\
Laparoscópicaa & & \\
\hline & & \\
\hline
\end{tabular}

Tabla 2. Análisis bivariado de Infección de herida operatoria.

\begin{tabular}{lccc} 
& \multicolumn{3}{c}{ Infección de herida operatoria } \\
& RP & IC & P \\
\hline Sexo & 0,98 & $0,67-1.44$ & 0,922 \\
Grado militar & 1,292 & $0,89-1,88$ & 0,18 \\
Hipertensión Arterial & 1,34 & $0,93-1,95$ & 0,12 \\
\hline Diabetes mellitus & 1,49 & $1,03-2,18$ & 0,035 \\
\hline Neoplasia maligna & 1,06 & $0,67-1,65$ & 0,813 \\
Obesidad & 0,94 & $0,63-1,39$ & 0,766 \\
\hline Tipo de cirugía & 4,63 & $2,89-7,42$ & $\mathrm{p}<0,05$ \\
\hline Técnica quirúrgica & 0,24 & $0,13-0,43$ & $\mathrm{p}<0,05$ \\
\hline
\end{tabular}


Tabla 3. Análisis multivariado de infección de herida operatoria.

\begin{tabular}{lccc} 
& \multicolumn{3}{c}{ Infección de herida operatoria } \\
& RP & IC & p \\
\hline Diabetes mellitus & 1,33 & $0,98-1,79$ & 0,065 \\
Tipo de cirugía & 4,04 & $2,55-6,40$ & $\mathrm{p}<0,05$ \\
Técnica quirúrgica & 0,29 & $0,17-0,52$ & $\mathrm{p}<0,05$ \\
\hline
\end{tabular}

\section{DISCUSIÓN}

El envejecimiento es una fase de nuestro ciclo vital, que cursa con características propias algunas más agradables que otras que se manifiestan de manera progresiva de acuerdo con factores extrínsecos e intrínsecos. Estimaciones del Instituto Nacional de Estadística e Informática (INEI) indican que al año 2018 la población adulta mayor representa el 11,9 $\%$ de la población general; hallándose un incremento poblacional del $1 \%$ en los últimos 10 años ${ }^{7}$. Esto constituye un grupo poblacional cuyo interés no puede desligarse de la calidad de vida, ni de la identificación de factores que pudieran influir en ellos, ya sea en el extrahospitalario o en el intrahospitalario. En el caso específico del pos operatorio, la población adulta mayor presenta ciertas características clínicas que las predisponen a situaciones como las infecciones de sitio operatorio ${ }^{8}$.

En nuestro estudio se muestra que un $41.05 \%$ de la población adulta mayor con diabetes mellitus presento ISO siendo una cifra comparable a la de una revisión sistemática donde se determina que la probabilidad de generar ISO es mayor al $40 \%{ }^{9}$. Por otro lado se encuentra un $32 \%$ de frecuencia de obesidad e ISO, datos cercanos a los reportados en un estudio de cohortes donde se determina una frecuencia del 40 a $60 \%{ }^{10}$.

En relación a los factores asociados a ISO, se encontró que la probabilidad de que se produzca una ISO después de una cirugía de emergencia fue del 4,04 en comparación con la cirugía programada, lo cual es respaldado por otros estudios con hallazgos similares puesto que en cirugías no programadas, las condiciones pre hospitalarias no pueden controlarse adecuadamente y en muchos casos urge la intervención quirúrgica inmediata ante los riesgos para la vida ${ }^{11,12,13}$. Por otro lado, se determina que la Cirugía de Técnica laparoscópica tiene 0,29 veces la probabilidad de ISO en comparación con la técnica abierta; lo que demostraría que el riesgo de ISO es menor cuando se practica una cirugía laparoscópica. En diferentes estudios se ha demostrado que la técnica laparoscópica tiene una menor tasa de infecciones gracias al menor grado de exposición a gérmenes ${ }^{14,15}$. En ambos casos, si bien la literatura precedente establece estos parámetros sin hacer diferencias entre pacientes adultos mayores y población en general, estudios adicionales deberían enfocarse en este grupo etáreo ${ }^{16,17}$.

Cuando se analizó en la muestra la comorbilidad DM, inicialmente resultó significativa en el análisis bivariado, sin embargo en el multivariado no resultó significativa, esto se debe a que posiblemente es un factor confusor. Esto puede deberse a un tamaño insuficiente de la muestra que impidió una adecuada comparación. Estudios adicionales deben plantearse para generar mayores niveles de evidencia relacionados a comorbilidades e ISO.

\section{LIMITACIONES DEL ESTUDIO}

El estudio es unicéntrico, así mismo el número de casos se compensa con los controles.

Este tipo de estudio solo nos permite detectar posibles asociaciones, y generar futuras hipótesis de relación causa efecto, en comparación con estudios multicéntricos, longitudinales y prospectivos.

\section{CONCLUSIÓN}

La técnica operatoria laparoscópica disminuye la probabilidad de ISO, y la cirugía de emergencia aumenta la probabilidad de ISO en pacientes adultos mayores.

Contribuciones de autoría: Los autores participaron en la concepción, recolección de información, redacción y aprobación de la versión final del artículo.

Financiamiento: Autofinanciado.

Conflicto de intereses:Los autores declaran no tener conflicto de interés en la publicación de este artículo. Los autores declaran no tener conflicto de interés en la publicación de este artículo.

Recibido: 20 de enero del 2019

Aprobado: 07 de junio del 2019

Correspondencia: Lucía Fernanda Hidalgo Vizarreta.

Dirección: Avenida S/N, Av. República de Venezuela, Bellavista, lima-Perú.

Teléfono: +51994377166

Correo: luciahidalgo94@gmail.com 


\section{REFERENCIAS BIBLIOGRÁFICAS}

1. Alvarado García, Alejandra; Salazar Maya, Ángela. Análisis del concepto de envejecimiento. Gerókomos. [Internet]. 2014 [citado 4 de diciembre de 2018]. 25(2): 57-62 Disponible en: http://scielo.isciii.es/ pdf/geroko/v25n2/revision1.pdf

2. López-Soto A. Infecciones en el anciano. Medicina Integral. 2012. 40(10): 476-84. Disponible en: https://www.elsevier.es/es-revistamedicina-integral-63-pdf-13041788.

3. Bagdasarian N, Schmader KE, Kaye KS. The Epidemiology and Clinical Impact of Surgical Site Infections in the Older Adult. Curr Transl Geriatr Exp Gerontol Rep. 1 de septiembre de 2013;2(3):159-66.

4. Horasan ES, Dağ A, Ersoz G, Kaya A. Surgical site infections and mortality in elderly patients. Med Mal Infect. octubre de 2013;43(10):417-22.

5. Kaye KS, Sloane R, Sexton DJ, Schmader KA. Risk Factors for Surgical Site Infections in Older People. J Am Geriatr Soc. 1 de marzo de 2006;54(3):391-6.

6. Agodi A, Quattrocchi A, Barchitta M, Adornetto V, Cocuzza A, Latino R, et al. Risk of surgical site infection in older patients in a cohort survey: targets for quality improvement in antibiotic prophylaxis. Int Surg marzo de 2015;100(3):473-9.

7. Instituto Nacional de Estadística e Informática [Internet]. [citado 6 de diciembre de 2018]. Disponible en: https://www.inei.gob.pe/bibliotecavirtual/boletines/ninez-y-adulto-mayor/2/

8. Beorlegui JS, Laguna EM, Sancho AA, Marcos NMD. Colecistectomía laparoscópica en octogenarios. 2008; 23(3):136-145. Disponible en: http://www.scielo.org.co/pdf/rcci/v23n3/v23n3a3.pdf

9. Centro Médico Teknon. Cirugía de urgencias [Internet]. 2018 [citado 5 de diciembre de 2018]. Disponible en: http://www.teknon.es/es/ especialidades/diez-caballero-alonso-alberto/cirugia-urgencias
10. Londoño F Á, Morales E J, Murilla B M. Características epidemiológicas y factores de riesgo relacionados con la infección en el sitio operatorio en procedimientos de cirugía general. Rev Chil Cir. diciembre de 2011;63(6):559-65.

11. Jiménez MF, Moore JH, Quintero G, Lerma C, Nieto JA, Fajardo R. Guía para la prevención de la infección del sitio operatorio (ISO). 2015;5:24

12. Cirugía abierta general [Internet]. [citado 5 de diciembre de 2018]. Disponible en:https://www.bbraun.es/es/productos-y-terapias/cirugiaabierta-general.html

13. SHEA. Diabetes Identified as a Risk Factor for Surgical Site Infections - SHEA [Internet]. 2015 [citado 3 de diciembre de 2018]. Disponible en: https://www.shea-online.org/index.php/journal-news/press-room/ press-release-archives/432-diabetes-identified-as-a-risk-factor-forsurgical-site-infections

14. PERÚ Instituto Nacional de Estadística e Informática [Internet] [citado 6 de diciembre de 2018]. Disponible en: https://www.inei.gob. pe/biblioteca-virtual/boletines/ninez-y-adulto-mayor/2/

15. Introducción a la técnica laparoscópica diagnóstica: Indicaciones preparación y pasos previos. [Internet]. [citado 5 de diciembre de 2018] Disponible en: https://www.researchgate.net/publication/305700493_ Introduccion_a_la_tecnica_laparoscopica_diagnostica_Indicaciones preparacion_y_pasos_previos

16. Gould LJ, Fulton AT. Wound Healing in Older Adults. 2015;3.

17. Pilco GTH. Factores asociados a complicaciones postoperatorias en el abdomen agudo quirúrgico del paciente adulto mayor. Hospital Regional Honorio Delgado- 2017. :55.
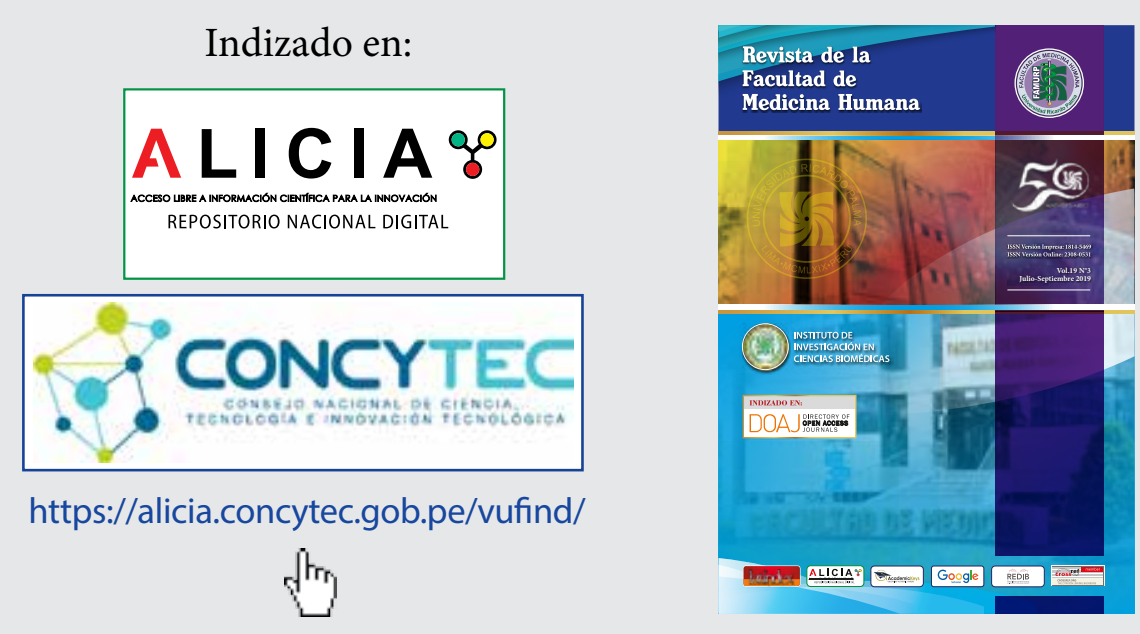